Thorax (1974), 29, 589.

\title{
Idiopathic calcified myocardial mass
}

\author{
DAVID PATTERSON ${ }^{1}$, DEREK GIBSON, \\ R I C A D O GOMES, LAW SON M D O A L D, \\ ECKHARDT OLSEN, JOHN PARKER, a nd \\ D O N A D ROSS
}

National Heart Hospital and Brompton Hospital, London

\begin{abstract}
Patterson, D., Gibson, D., Gomes, R., McDonald, L., Olsen, E., Parker, J.; and Ross; D. (1974). Thorax, 29, 589-594. Idiopathic calcified myocardial mass. Myocardial calcification can be subdivided into three groups-metastatic, dystrophic or an extension inwards from the pericardium. This case in which the calcified myocardial mass was initially delineated by radiography and by echocardiography and subsequently removed does not fit into any subdivision and has been termed idiopathic.
\end{abstract}

Myocardial calcification has been subdivided by Finestone and Geschickter (1949) and Gore and Arons (1949) into three groups-metastatic, dystrophic or an extension inwards from the pericardium. In the metastatic group hypercalcaemia is usually present and calcium salts are deposited in tissues which were previously normal. The dystrophic calcification is related to the laying down of calcium salts in dead, degenerated or devitalized myocardial tissue.

This case in which the calcified mass was successfully removed does not satisfactorily fit into any subdivision and it has therefore been termed idiopathic.

\section{CASE REPORT}

Miss M.D. aged 52 first developed symptoms at the age of 42 when she was suddenly awakened by breathlessness and palpitations. She subsequently felt tired and lacked energy; there was no associated chest discomfort and no expectoration of sputum. She was found to be in atrial fibrillation. Her symptoms improved spontaneously although she remained tired. At age 48 she suddenly developed a right lower nasal visual field defect that has persisted. No other neurological deficit was noted. At age 50 she noticed increasing shortness of breath which improved with diuretic therapy but one year later she became increasingly short of breath on exertion and orthopnoeic, and developed paroxysmal nocturnal dyspnoea. Her ankles and abdomen swelled and she again noted palpitations. No chest discomfort was associated with these symptoms. Digoxin, frusemide, and anticoagulants were started with some improvement in her symptoms.

'Present address: Royal Free Hospital, Gray's Inn Road, London WC1
Four months later she developed a saddle embolus while still on anticoagulant treatment; this necessitated bilateral femoral thrombectomy.

Her past medical history revealed that diphtheria was questioned at the age of 23 but never substantiated. She had no history of rheumatic fever.

Her father died aged 72 of a myocardial infarction and her mother died aged 72 of a cerebrovascular accident. One brother aged 50 had had a myocardial infarction; five sisters are alive and well.

She gave up smoking cigarettes at the age of 51 . Her menopause occurred at age 49. She had never been abroad.

Physical examination revealed her to be in no acute distress. She was normotensive. There was no arcus and no xanthomata. There was no evidence of heart failure. There were no pulses palpable below the femoral arteries but there were no trophic skin changes. The apex beat was not displaced. The heart sounds and murmurs suggested mild aortic regurgitation and mild mitral valve disease. The remainder of the physical examination was normal.

INVESTIGATIONS An electrocardiogram (Fig. 1) showed atrial fibrillation with a controlled ventricular rate. The QRS axis and QRS duration $(0.10 \mathrm{sec})$ were normal. Voltage criteria of left ventricular hypertrophy were present with ST segment and $T$ wave abnormalities. There was no evidence of old myocardial infarction.

A chest radiograph (Fig. 2) showed enlargement of the left atrium and some pulmonary venous congestion. There was dense intracardiac calcification situated in the interventricular septum and remote from the mitral valve ring. There was no pericardial calcification. 


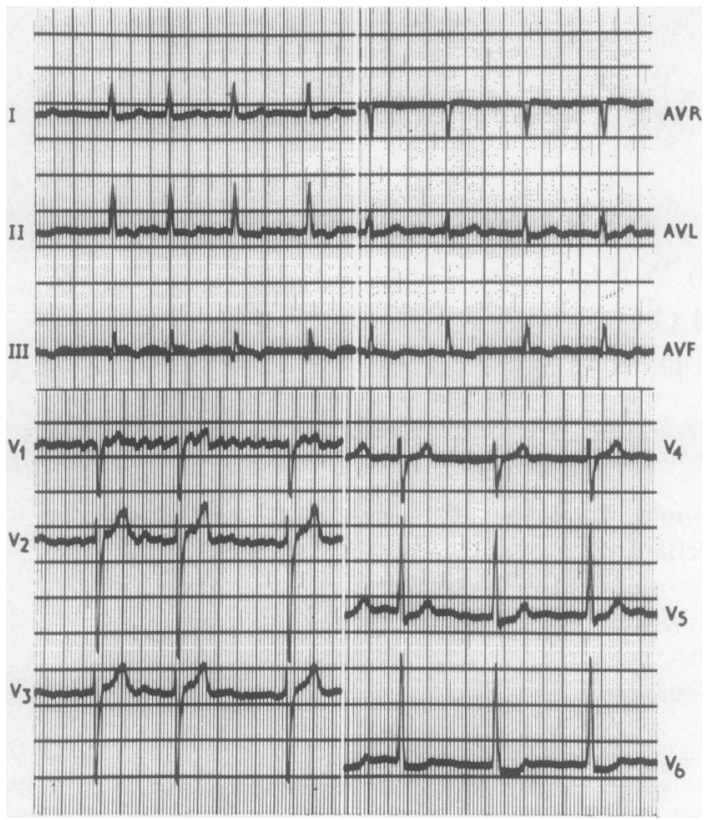

FIG. 1. Preoperative electrocardiogram showing atrial fibrillation and normal $Q R S$ axis and duration, left ventricular hypertrophy, and $S T$ segment and $T$ wave abnormalities. Patient was taking digoxin.

Radiographs of the hands, feet, lumbar spine, and kidneys revealed no evidence of abnormal calcification.

Laboratory tests showed haemoglobin, white cell count, erythrocyte sedimentation rate, urea and electrolytes, uric acid, liver function tests, serum proteins, urine examination, serum calcium, phosphorus and alkaline phosphatase, and a 24-hour urinary calcium all to be normal. The Wassermann and hydatid complement fixation tests were negative.

Serum cholesterol was $202 \mathrm{mg} / 100 \mathrm{ml}$, serum triglycerides were $222 \mathrm{mg} / 100 \mathrm{ml}$, and lipoprotein electrophoresis demonstrated a normal beta band and an increased pre-beta band consistent with a Fredrickson type IV hyperlipoproteinaemia (Fredrickson, Levy, and Lees, 1967).

Echocardiography (Fig. 3) demonstrated the left ventricular cavity to be enlarged. Movements of the interventricular septum and posterior wall were normal. The two septal echoes were not abnormally separated. An abnormal echo was demonstrated $2-3 \mathrm{~cm}$ thick about $1 \mathrm{~cm}$ behind the septum in the left ventricular cavity. Its movement was parallel to that of the posterior left ventricular wall and aortic root and the reverse of that of the septum.

Echocardiography of the mitral valve (Fig. 4) demonstrated impaired diastolic mobility of the anterior cusp. The cusp was not thickened. This was considered to be consistent with rheumatic mitral valve disease.
CARDIAC CATHETERIZATION There were normal right heart pressures with a mean pulmonary arterial wedgo pressure of $16 \mathrm{mmHg}$ rising to $25 \mathrm{mmHg}$ aftey exercise. The end-diastolic indirect mitral valve gradient was $0 \mathrm{mmHg}$ at rest rising to $10 \mathrm{mmHg}$ after exercise. No systolic aortic valve gradient was. present. Left ventricular end-diastolic pressure waP $15 \mathrm{mmHg}$ rising to $32 \mathrm{mmHg}$ after angiography.

Angiography The left ventriculogram revealed thaథ contractility of the left ventricle was severely im paired by the calcified mass, and numerous lobulate calcified excrescences encroached into the lefte ventricular cavity. The intracardiac calcification was confirmed to have its main concentration towards the apex but extended into the interventricular septum $\mathrm{O}_{\odot}$ Slight mitral regurgitation was present.

Selective coronary arteriography revealed normat coronary arteries.

The aortogram confirmed mild aortic regurgitation

OPERATION A median sternotomy was performed i March 1972. The left atrium was enlarged and the intracardiac mass was readily palpable in the dia phragmatic and lateral walls of the ventricle. Openings the left atrium revealed a mitral orifice of about $1 \mathrm{~cm}^{2}$ which was of rheumatic appearance. The mas? in the ventricle was then approached through the diaphragmatic surface of the heart by an incisiote parallel to the posterior descending artery. The calco fied mass was located 3-4 mm from the epicardiun and had irregular projections extending into a health $\overrightarrow{\mathscr{F}}$ looking muscle. It was found to extend into the lefe ventricular cavity and up the diaphragmatic surfaceof the ventricle laterally, just across onto the septuri about $2 \mathrm{~cm}$ below the aortic valve and about $2 \mathrm{~cm}$ from the mitral valve. It was enucleated by shar dissection (Fig. 5). The mitral valve was replaced with a Starr-Edwards prosthesis and the ventriculotomy was closed with Teflon support.

The immediate postoperative course was uneventfut and there was a marked symptomatic improvement The postoperative electrocardiogram showed no in crease in the QRS duration but demonstrated a slight intraventricular conduction disturbance. Chest radios graphs showed no change in heart size and confirme that virtually all the visible myocardial calcified mass had been removed. An echocardiogram (Fig. 69 showed that the intracavitary echoes were no longer present and that the left ventricular cavity remaine large. Septal movement was abnormal, which is characteristic of a normally functioning mitral Starr? Edwards valve and reflects a reduced rate of earks diastolic left ventricular filling.

Seven months after the operation the patient suddenly collapsed and died. No necropsy waso performed.

PATHOLOGY The specimen submitted for examinatio $\mathbb{B}$ consisted of three pieces of calcified tissue, measuring $1.6,1.9$, and $0.9 \mathrm{~cm}$ in the longest diameter respecs 




(a)

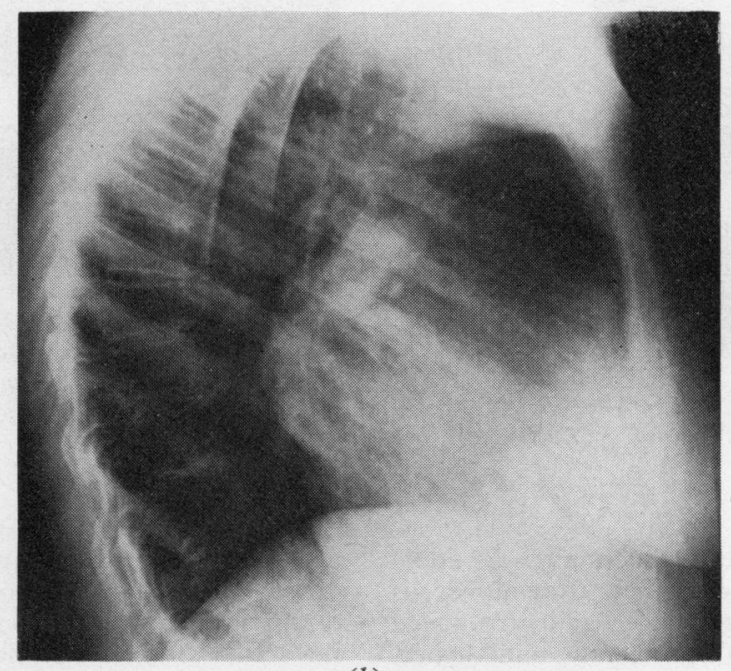

(b)

FIG. 2. Chest radiographs $P A(a)$ and lateral (b) showing an enlarged left atrium, pulmonary venous congestion, and dense intracardiac calcification. 


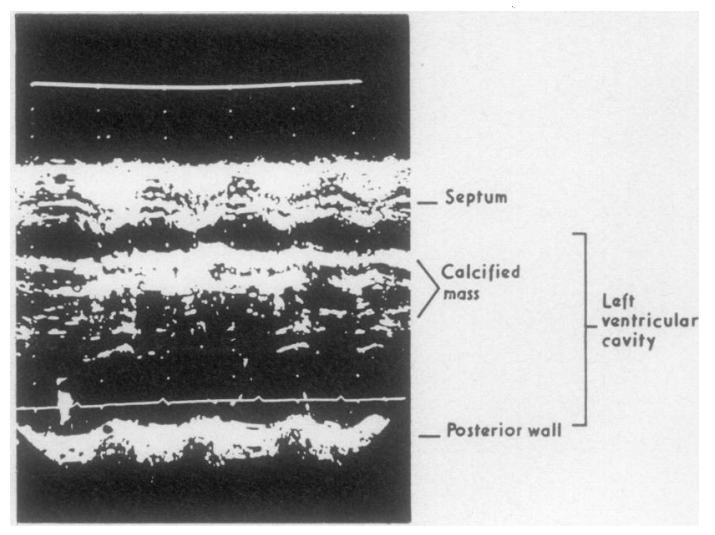

FIG. 3. Preoperative echocardiogram showing an enlarged left ventricular cavity with normal movement of the posterior wall and interventricular septum. Abnormal echo demonstrated $1 \mathrm{~cm}$ behind septum in the left ventricular cavity.



FIG. 4. Preoperative echocardiogram of the mitral valve showing impaired diastolic mobility of the anterior cusp.

tively. In two of these pieces myocardial tissue could be identified with the naked eye. There were numerous large areas of calcification embedded and surrounded by myocardial fibres which apart from mild hypertrophic changes showed no abnormality (Fig. 7). In addition to this, an occasional area of fibrous tissue rich in capillaries was present. Surrounding the calcific areas a scanty chronic inflammatory infiltrate, patchily distributed, was present.



FIG. 5. Calcified mass removed at surgery.
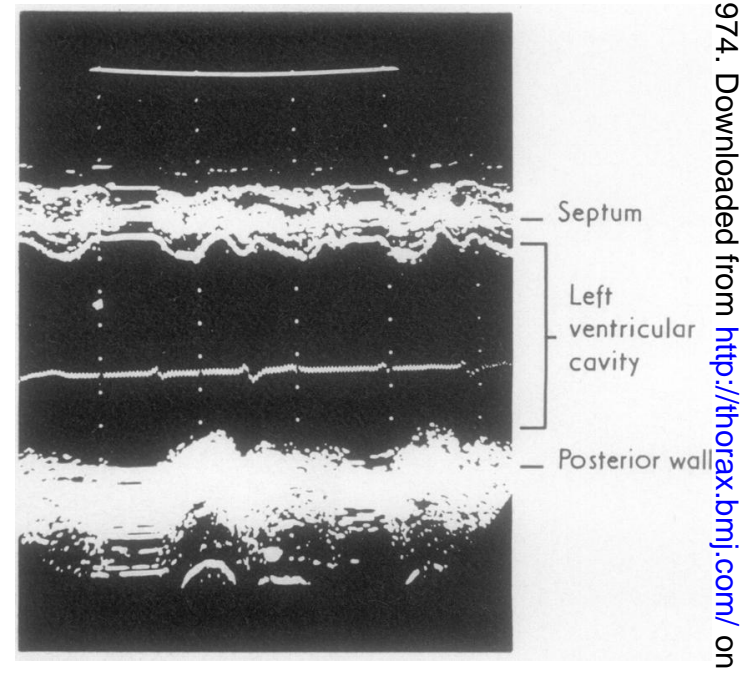

FIG. 6. Postoperative echocardiogram showing the absence of the intracavitary echoes. Left ventricula?. cavity remained large, and abnormal septal move $N$ ments were demonstrated.

The mitral valve cusp showed thickening pre dominantly by collagen tissue particularly at the enfe distal to the line of closure of the valve leaflets. Some recent fibrin was also present on the deformed atriaF surface of the valve leaflets. Vascularity was slightly응 increased, compatible with a rheumatic aetiology.

A portion of the posterior mitral valve leaflet was? available for serial sectioning. Every tenth section $\mathbb{D}$ cut at 5 microns thickness, was examined. There was no calcification in any of the sections examined. 


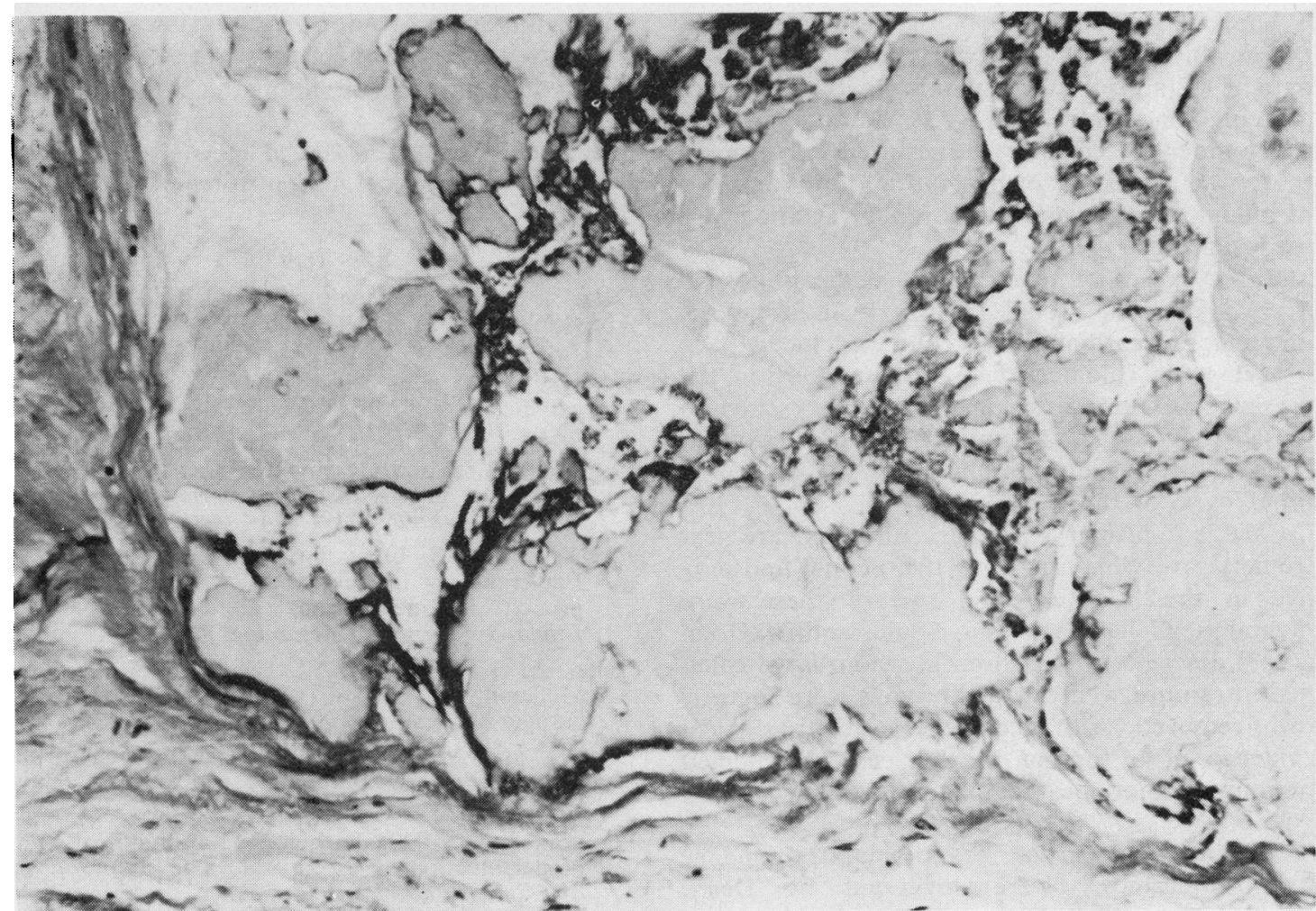

FIG. 7. Photomicrograph of part of the intracardiac calcific mass showing displacement of surrounding myocardial tissue (Haematoxylin and eosin $\times 190$ ).

\section{DISCUSSION}

The clinical features in the present case were not helpful in establishing the diagnosis, since systemic emboli, increasing breathlessness, and fluid retention might all have been due to mitral valve disease with associated atrial fibrillation. Chest radiography was of value in demonstrating intramyocardial calcification although its precise delineation required left ventriculography. The presence of the mass within the left ventricular cavity was also demonstrable by echocardiography and shown to be distinct from the mitral valve, with movement parallel to the posterior wall rather than to the septum. Although echocardiography is now a standard method of diagnosing left atrial tumours causing interference with mitral valve function, this appears to be the first time that the method has been used to demonstrate an intraventricular tumour. Although calcification extended into the lower part of the septum, there does not seem to have been involvement of the conduction system, since the QRS duration was normal.
Mitral ring calcification with involvement of adjoining myocardium is well recognized in 'older' women (Pomerance, 1970). The primary lesion may be due in part to calcification of organized thrombus in the subvalvar angle of the mitral ring. This mechanism is unlikely in this patient as the calcification was remote from the mitral valve ring and there was no histological evidence of calcification in the available sections of the mitral valve ring which were removed at surgery.

It has been suggested by Ernstene and Hazard (1951) that deposition of calcium may occur during the healing phase of a severe toxic or septic myocarditis. The patient had rheumatic involvement of the mitral and aortic valves, and it can be postulated that a severe myocarditis was associated with her rheumatic fever. However, scanty inflammatory infiltrate is not uncommon around areas of calcification, and elsewhere the myocardium was normal; this suggests that a past myocarditis is unlikely. The size that the calcified mass attained also makes a 'dystrophic' mechanism unlikely. 
The commonest cause of dystrophic calcification in this country is coronary heart disease. The patient's family history, smoking habit, and lipoprotein abnormality are 'risk factors' in the development of coronary heart disease. However, there was no history of myocardial infarction nor of angina pectoris and the coronary arteriograms were normal.

Rarer causes of myocardial calcification such as hypokalaemia, renal failure, hydatid disease and endomyocardial fibrosis can be excluded.

Metastatic calcification is also unlikely in the presence of normal serum and urinary calcium levels, the absence of any destructive lesion of bone, and the absence of abnormal calcification in other parts of the body.

Calcified primary cardiac tumours are extremely rare. Shapiro et al. (1963) could find only five in the literature and two of these were myxomas. Geha, Weidman, Soule, and McGoon (1967) describe two children in whom large calcified intramural ventricular fibromas were successfully removed. Although there is no histological evidence of a tumour in this case, a calcified tumour cannot be completely excluded as a possibility.

Calcified endocardial granulomata secondary to histoplasmosis were suggested by Dean, Pamukcoglu and Roberts (1969) to be the cause of some small calcified nodules found in the right ventricular cavity of a male patient. There was no evidence of histoplasmosis in this patient, however.

The cause of this calcified myocardial mass re- mains unknown. It does not fit into the three recognized subdivisions of myocardial calcification $\frac{\bar{\sigma}}{\bar{N}}$ and is therefore termed 'idiopathic'.

The authors thank Dr. J. K. Finlayson for referring कै this patient and for permission to report the case.

\section{REFERENCES}

Dean, D. C., Pamukcoglu, T., and Roberts, W. C. $\overrightarrow{\vec{x}}$ (1969). Rocks in the right ventricle. American Journal of Cardiology, 23, 744.

Ernstene, A. C. and Hazard, J. B. (1951). Extensive calcification of the myocardium. Circulation, 3, $\infty$ 690.

Finestone, A. J. and Geschickter, C. F. (1949). Bone $\stackrel{\circ}{?}$ formation in the heart. American Journal of $\vec{B}$ Clinical Pathology, 19, 974.

Fredrickson, D. S., Levy, R. I., and Lees, R. S. (1967). Fat transport in lipoproteins-an integrated ap- $\frac{\mathbb{D}}{3}$ proach to mechanisms and disorders. New England Journal of Medicine, 276, 273.

Geha, A. S., Weidman, W. H., Soule, E. H., and $\vec{\theta}$ McGoon, D. C. (1967). Intramural ventricular cardiac fibroma. Circulation, 36, 427.

Gore, I. and Arons, W. (1949). Calcification of the myocardium. A pathologic study of thirteen cases. Archives of Pathology, 48, 1.

Pomerance, A. (1970). Pathological and clinical study of calcification of the mitral valve ring. Journal of Clinical Pathology, 23, 354.

Shapiro, J. H., Jacobson, H. G., Rubinstein, B. M., 윽 Poppel, M. H., and Schwedel, J. B. (1963). Calcification of the Heart. Thomas, Springfield, Illinois.

Requests for reprints to: Dr. David Patterson, Royal Free Hospital, Gray's Inn Road, London WC1. 